\title{
Fatal Ruptured Blood Blister-like Aneurysm of Middle Cerebral Artery Associated with Ehlers-Danlos Syndrome Type VIII (Periodontitis Type)
}

\author{
Ulaş Cıkla ${ }^{1} \quad$ Alireza Sadighi $^{1} \quad$ Andrew Bauer ${ }^{1} \quad$ Mustafa K. Başkaya ${ }^{1}$ \\ ${ }^{1}$ Department of Neurosurgery, University of Wisconsin-Madison, \\ Madison, Wisconsin, United States \\ Address for correspondence Mustafa K. Başkaya, MD, Department of \\ Neurological Surgery, University of Wisconsin-Madison, School of \\ Medicine, CSC, K4/822, 600 Highland Avenue, Madison, WI 53792, \\ J Neurol Surg Rep 2014;75:e210-e213. \\ United States (e-mail: m.baskaya@neurosurgery.wisc.edu).
}

\begin{abstract}
Objective To illustrate a unique instance of Ehlers-Danlos syndrome type VIII (EDS VIII) with blood blister-like aneurysm of the middle cerebral artery.

Design This is a single patient case report.

Setting University of Wisconsin-Madison Hospital.

Participants A 42-year-old woman with diagnosis of EDS VIII presented with a sudden onset severe headache and altered mental status. She was diagnosed with Hunt and Hess grade IV subarachnoid hemorrhage. Angiography demonstrated a blood blister-

Keywords

- Ehlers-Danlos syndrome

- blood blister-like aneurysm

- periodontal disease

- internal carotid artery like aneurysm of the left middle cerebral artery. After an unsuccessful coiling attempt in another facility, the patient was operated on with the intention to perform extracranial to intracranial bypass and trapping of the diseased segment of the artery.

Results The patient's neurologic condition remained poor after surgery. On postoperative day 2 , her neurologic examination unchanged, and care was withdrawn per the family's request.

Conclusions Individuals with EDS VIII may be at risk for catastrophic vascular events.
\end{abstract}

\section{Introduction}

The Ehlers-Danlos syndromes (EDS) are an inherited heterogeneous group of connective tissue disorders, principally characterized by tissue fragility, the hypermobility of joints, and the hyperextensibility of the skin. ${ }^{1}$ In the Villefranche nosology, these disorders are classified into six major subtypes (and some rare subtypes) based on clinical, biochemical, and molecular characteristics. ${ }^{2}$ The major types are classic, hypermobility, kyphoscoliotic, vascular, dermatosparaxis, and arthrochalasia types; one of the rare subtypes is the periodontitis type, also called EDS type VIII (EDS VIII).

EDS VIII is an autosomal dominant disorder characterized by gingival recession and periodontal disease, leading to precocious dental loss and distinctive pigmentary scarring of the anterior surfaces of the lower legs, as well as the manifestations it shares with other forms of EDS. ${ }^{3-5}$ It has not been previously associated with catastrophic vascular complications or hollow viscous rupture that arise in EDS IV. We report a fatal intracerebral aneurysm in a patient with EDS VIII.

\section{Case Report}

The proband was initially evaluated when the patient was 13 years old. At that time, she presented with frequent bruising, fragility of the skin over the anterior aspects of her tibiae (over one of which she experienced an avulsion laceration), and gingival retraction with alveolar absorption. Because of periodontitis, all of her teeth had either been lost spontaneously or had been extracted by the time she was 19 years old. Scars in her shins were obvious by her teenage years (-Fig. 1). According to her history, the areas over her tibiae episodically swelled for prolonged periods, following received

January 28, 2014

accepted

May 27, 2014

published online

August 11, 2014
DOI http://dx.doi.org/

10.1055/s-0034-1387185. ISSN 2193-6358. (c) 2014 Georg Thieme Verlag KG
Stuttgart · New York

License terms

(c) $(1) \$$ 


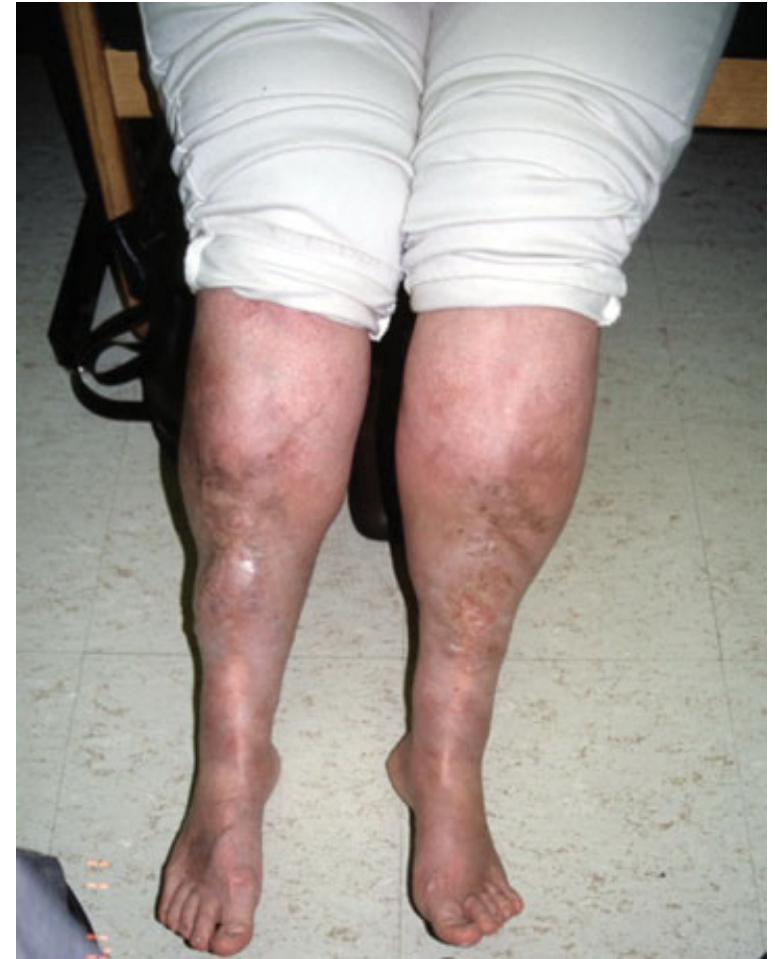

Fig. 1 Photograph demonstrating bilateral hyperpigmented scarring over the shins (photo courtesy of Richard M. Pauli).

which there was resolution into persistent hyperpigmented lesions. Additionally, her history was positive for easy bruisability, but she did not have either joint hyperextensibility or markedly hyperextensible skin by history. She had one normal pregnancy with an uncomplicated vaginal delivery. No complications related to hollow viscous or large vessels had arisen prior to the time of her genetics evaluation. The family history was negative for features suggestive of EDS.

She was assessed through the Clinical Genetics Center at the University of Wisconsin at 30 years of age. Examination confirmed the presence of mildly unusual facial features including shallow orbits and midfacial hypoplasia, edentulous state, incidental absent uvula secondary to operative removal for obstructive apnea, soft but not hyperextensible skin, dramatic pigmentary scarring over the tibiae (- Fig. 1), and mild joint hyperextensibility. A diagnosis of EDS VIII was made, but because of the known phenotypic overlap between this and EDS IV, biochemical and genetic analyses of collagens from skin fibroblasts were completed (in the laboratory of Peter Byers) that demonstrated normal type III collagen sequence and expression.

At age 42 , she presented with a sudden onset severe headache and altered mental status and was admitted to another facility. Noncontrast computed tomography (CT) of the head revealed a subarachnoid hemorrhage (SAH) with an intraventricular and intraparenchymal hemorrhage, and clinically, she was diagnosed with Hunt and Hess grade IV SAH. ${ }^{6,7}$ Digital subtraction angiography demonstrated a blood blister-like aneurysm of the left middle cerebral artery (MCA) in the M1 segment (-Fig. 2).

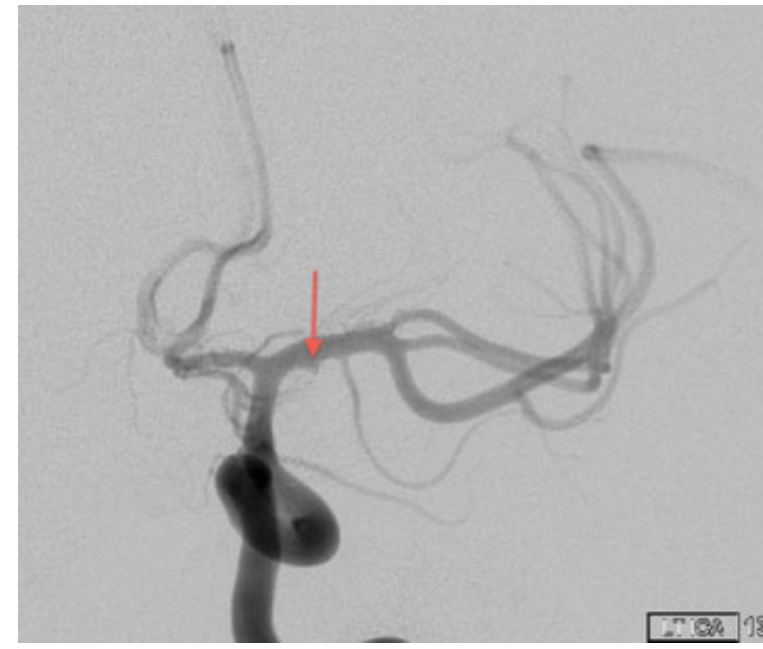

Fig. 2 Digital subtraction angiography demonstrating small aneurysm (arrow) at nonbranching side in the M1 segment of the left middle cerebral artery. These features, such as small size and occurrence at the nonbranching point of the parent artery, indicate this is not a saccular aneurysm but rather a blood blister-like or dissecting type aneurysm.

A subsequent attempted coiling procedure was unsuccessful at that facility. Shortly thereafter, she developed dilated pupils, and further imaging demonstrated rebleeding with mass effect on the left hemisphere. A decompressive craniectomy was performed. During the post-craniectomy state, her examination improved slightly to the point that her pupils were reactive and she had intact brainstem reflexes. She was transferred to our facility for definitive treatment. On arrival, she was intubated and had bilaterally reactive pupils and positive cough and corneal reflex. She had no response to painful stimuli on the right upper and lower extremities but some minimal uncharacterizable flicker movements on the left upper extremity. After obtaining a repeat CT ( - Fig. 3A, B) that demonstrated continuous bleeding from the aneurysm, she was taken to the operating room emergently with the intention to perform extracranial to intracranial bypass and trapping of the diseased segment of the artery. Her large scalp flap was quite tense. Upon reexploration, it was noted that the brain was extremely swollen and tight, and there was bleeding coming from the sylvian fissure and pretemporal region. The sphenoid wing was drilled quickly, and the left sylvian fissure was dissected. It was also noted that, overall, all the small arteries were fragile. The left internal carotid artery (ICA), anterior cerebral artery, and distal MCA were identified and isolated. Temporary clips were placed on the ICA and distal M1 segment of MCA to stop bleeding. Extreme difficulty was encountered in doing all these life-saving maneuvers due to her herniating brain. Finally, the proximal M1 segment of the left MCA, which was the diseased segment of the artery, was isolated and trapped. It was clear that a vessel sacrifice was the only possible lifesaving procedure. Permanent clips were placed onto the MCA. There was no clear aneurismal wall but, instead, a fibrin clot and tear in the artery, indicating this was a so-called blood blister-like aneurysm. Although the radial artery in the arm was prepared for a possible bypass 


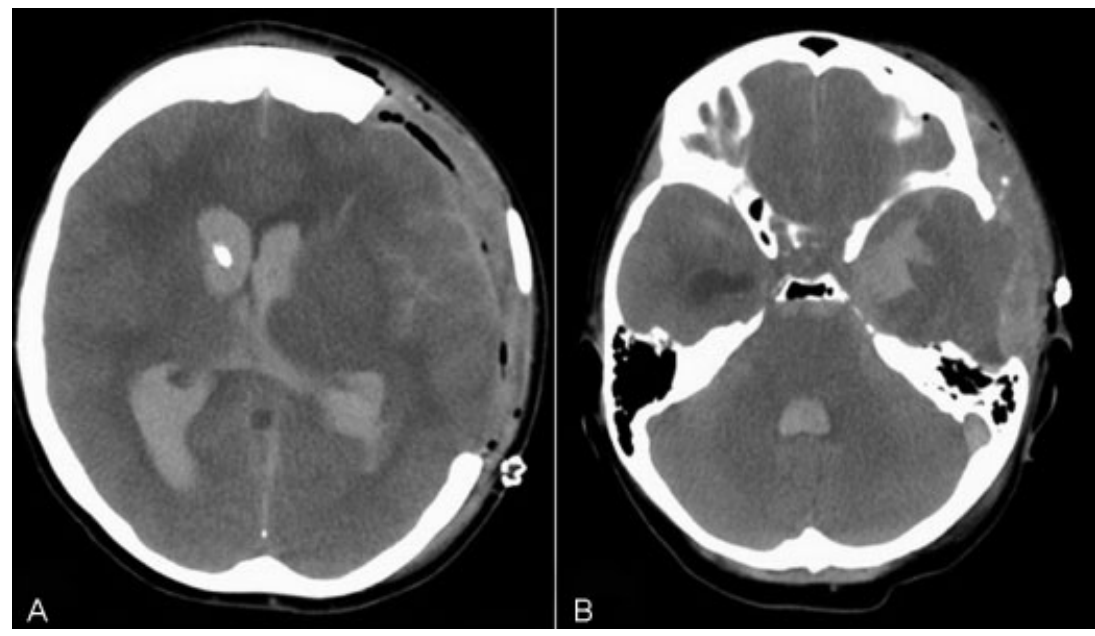

Fig. 3 (A, B) Noncontrast computed tomography scans of the head demonstrating subarachnoid, intraparenchymal, and intraventricular hemorrhages. Note the craniectomy defect and brain herniation through this defect.

procedure, due to difficulties with brain swelling and herniation, it was deemed bypass would not be possible.

Her neurologic condition remained poor after surgery. On postoperative day 2, her neurologic examination was unchanged, and care was withdrawn per the family's request.

\section{Discussion}

EDS VIII is characterized particularly by hyperpigmented scarring of the pretibial areas, variable bruisability and joint hypermobility, and generalized periodontitis with early loss of teeth. ${ }^{3,8-11}$ It was first described by McKusick and was established as a distinct entity after the report of Stewart et al. ${ }^{5,12,13}$

The patient described here had features consistent with EDS VIII including both severe periodontal disease and characteristic pigmentary scarring over the shins. Furthermore, biochemical testing of skin fibroblasts did not demonstrate any change suggestive of EDS IV. Nonetheless, despite that reassuring finding, she ultimately developed a fatal intracranial aneurismal rupture. To posit that she actually had EDS IV, one would have to accept that all of the following were present and yet she had that form: (1) no demonstrable type III collagen abnormality; (2) coincidental development of severe, early periodontal disease; (3) development of pretibial pigmentary scarring usually considered to be distinctive for EDS VIII; and (4) absence of other features often seen in individuals with EDS IV. It seems far more likely, then, that this is the first reported instance of vascular fragility and intracranial aneurysm in a person with EDS VIII.

Aneurysms that do not occur at branch points may be saccular or blood blister-like. The latter are extremely difficult to treat by surgical or endovascular means because of their small size, very fragile walls, lack of an aneurysm neck, and tendency to avulse with minimal manipulation. ${ }^{13}$ The most common location of blister-like aneurysms is the anteromedial aspect of the supraclinoid ICA. They can be confused with ophthalmic or superior hypophyseal aneurysms and sometimes can involve a long segment of the supraclinoid ICA where many important branches arise. ${ }^{13}$ They are believed to be counterparts of the dissecting aneurysms of the vertebrobasilar circulation.

Intracranial aneurysms are, of course, one hallmark of EDS IV. ${ }^{14}$ Although the vast majority of individuals with clinical features of EDS IV have a demonstrable abnormality of type III collagen, there are reported instances in which apparently normal collagen studies have been present in some individuals who putatively had EDS IV (see, for example, Pope et al ${ }^{15}$ ). The diagnosis of EDS VIII in the patient described here must remain formally uncertain because there is no known molecular marker of EDS VIII and there is, in fact, some clinical overlap between these two forms, and considerable variability even of hallmark features in various forms of EDS. ${ }^{16,17}$

Several authors have emphasized that the collagen analysis can readily distinguish most forms of EDS IV from EDS VIII. $3,4,11,18,19$ Nonetheless, the type III collagen abnormality can be seen in patients with periodontitis in both types. Nelson and King described an 18-year-old woman with atrophic pretibial scars and absence of periodontitis as a EDS VIII. ${ }^{20}$ Furthermore, Pope et al described one patient with many features in common with the woman presented here: pretibial hyperpigmentary scarring and a lethal intracranial aneurysmal rupture. Despite the presence of normal type III collagen studies, they nonetheless posited that she had EDS IV. ${ }^{15}$ However, one could as easily suggest that this is an example of EDS VIII with vascular complications as we describe here.

Clinical overlap between type IV and type VIII makes diagnostic certainty challenging. ${ }^{16}$ This case is a cautionary tale. Even with normal type III collagen assessment, individuals with apparent EDS VIII may be at risk for vascular anomalies and life-threatening complications.

\section{Acknowledgments}

We thank Drs. Richard M. Pauli and Peter H. Byers for their help with genetic analysis and confirming the diagnosis of the patient. We would also like to thank Richard Pauli for his help in revising the manuscript. 


\section{Conflict of Interest}

The authors declare that the article content was composed in the absence of any commercial or financial relationships that could be construed as a potential conflict of interest.

\section{References}

1 Emery AEH, Rimoin DL, Sofaer JA. Ehlers-Danlos syndrome. In: Byers PH, Holbrook KA, Barsh GS, eds. Principles and Practice of Medical Genetics. Edinburgh, UK: Churchill Livingstone; 1983: 836-50

2 Beighton P, De Paepe A, Steinmann B, Tsipouras P, Wenstrup RJ; Ehlers-Danlos National Foundation (USA) and Ehlers-Danlos Support Group (UK). Ehlers-Danlos syndromes: revised nosology, Villefranche, 1997. Am J Med Genet 1998;77(1):31-37

3 Karrer S, Landthaler M, Schmalz G. Ehlers-Danlos type VIII. Review of the literature. Clin Oral Investig 2000;4(2):66-69

4 Reinstein E, Wang RY, Zhan L, Rimoin DL, Wilcox WR. EhlersDanlos type VIII, periodontitis-type: further delineation of the syndrome in a four-generation pedigree. Am J Med Genet A 2011; 155A(4):742-747

5 Stewart RE, Hollister DW, Rimoin DL. A new variant of EhlersDanlos syndrome: an autosomal dominant disorder of fragile skin, abnormal scarring, and generalized periodontitis. Birth Defects Orig Artic Ser 1977;13 0B 85-93

6 Fisher CM, Kistler JP, Davis JM. Relation of cerebral vasospasm to subarachnoid hemorrhage visualized by computerized tomographic scanning. Neurosurgery 1980;6(1):1-9

7 Hunt WE, Hess RM. Surgical risk as related to time of intervention in the repair of intracranial aneurysms. J Neurosurg 1968;28(1):14-20

8 Biesecker LG, Erickson RP, Glover TW, Bonadio J. Molecular and cytologic studies of Ehlers-Danlos syndrome type VIII. Am J Med Genet 1991;41(3):284-288

9 Dyne KM, Vitellaro-Zuccarello L, Bacchella L, Lanzi G, Cetta G. Ehlers-Danlos syndrome type VIII: biochemical, stereological and immunocytochemical studies on dermis from a child with clinical signs of Ehlers-Danlos syndrome and a family history of premature loss of permanent teeth. Br J Dermatol 1993;128(4):458-463

10 Mataix J, Bañuls J, Muñoz C, Bermejo A, Climent JM. Periodontal Ehlers-Danlos syndrome associated with type III and I collagen deficiencies. Br J Dermatol 2008;158(4):825-830

11 Moore MM, Votava JM, Orlow SJ, Schaffer JV. Ehlers-Danlos syndrome type VIII: periodontitis, easy bruising, marfanoid habitus, and distinctive facies. J Am Acad Dermatol 2006;55 (2, Suppl): S41-S45

12 McKusick VA. Heritable Disorders of Connective Tissue. 4th ed. Saint Louis, MO: Mosby, London, distributed by Kimpton; 1972

13 Başkaya MK, Ahmed AS, Ateş O, Niemann D. Surgical treatment of blood blister-like aneurysms of the supraclinoid internal carotid artery with extracranial-intracranial bypass and trapping. Neurosurg Focus 2008;24(2):E13

14 Schievink WI, Link MJ, Piepgras DG, Spetzler RF. Intracranial aneurysm surgery in Ehlers-Danlos syndrome type IV. Neurosurgery 2002;51(3):607-611; discussion 611-613

15 Pope FM, Nicholls AC, Narcisi P, et al. Type III collagen mutations in Ehlers Danlos syndrome type IV and other related disorders. Clin Exp Dermatol 1988;13(5):285-302

16 Hartsfield JK Jr, Kousseff BG. Phenotypic overlap of EhlersDanlos syndrome types IV and VIII. Am J Med Genet 1990; 37(4):465-470

17 Proske S, Hartschuh W, Enk A, Hausser I. Ehlers-Danlos syndrome -20 years experience with diagnosis and classification at the university skin clinic of Heidelberg. [in German]. J Dtsch Dermatol Ges 2006;4(4):308-318

18 Buckel T, Zaenglein AL. What syndrome is this? Ehlers-Danlos syndrome type VIII. Pediatr Dermatol 2007;24(2):189-191

19 Cunniff C, Williamson-Kruse L. Ehlers-Danlos syndrome, type VIII presenting with periodontitis and prolonged bleeding time. Clin Dysmorphol 1995;4(2):145-149

20 Nelson DL, King RA. Ehlers-Danlos syndrome type VIII. J Am Acad Dermatol 1981;5(3):297-303 